\title{
FRANZISKA ROOSEN
}

\section{ERZIEHUNG UND BILDUNG VON HUGENOTTEN IN BERLIN}

\author{
Das Lehrerseminar ${ }^{1}$
}

"Le bon Dieu ne parle pas l'Allemand ${ }^{2}$. Dieses Bonmot findet sich auf einer der ersten Seiten der Familienchronik, die der Hugenottennachfahre Gustav von Jordan zu Beginn des 20. Jahrhunderts verfaßte. Es macht auf amüsante Weise auf hugenottische Vorbehalte der deutschen Sprache gegenüber aufmerksam. Betrachtet man indes die Sprachpolitik der Französischen Kirche Brandenburg-Preußens im ausgehenden 18. und beginnenden 19. Jahrhundert, läßt sich dieser Ausspruch in ironischer Überspitzung auch auf all jene beziehen, die vor ihrer Kirche als gute Hugenotten dastehen wollten: „Ein Hugenotte spricht nicht Deutsch.»

$\mathrm{Zu}$ einer solchen Einschätzung führen die geradezu trotzigen Bemühungen der französischen Konsistorien, die Sprache der Ahnen unter den Gemeindemitgliedern lebendig zu halten. Herausragendes Motiv ihrer Sprachpolitik scheint die Angst gewesen zu sein, die durch das Potsdamer Edikt verbrieften Privilegien zu verlieren, wenn man sich von den Einheimischen nicht mehr deutlich unterscheiden würde ${ }^{3}$. Diese 1685 von den Hohenzollern zugestandenen Privilegien waren umfangreich: Sie gestatteten es den Hugenotten, in Kolonien mit eigener Kirche, eigener Verwaltung, eigener Rechtsprechung und eigener Schule zu leben. Aus diesem Blickwinkel betrachtet läßt sich die bewahrende Sprachpolitik der Französischen Kirche als Versuch charakterisieren, eine endgültige Assimilation ihrer Mitglieder an deren brandenburgisches Umfeld zu verhindern.

Welche Rolle in diesem Kontext der Schule zukam, soll im folgenden am Beispiel des hugenottischen Kantoren- und Lehrerseminars - der sogenannten

\footnotetext{
${ }^{1}$ Für kritische und beherzte Lektüre danke ich meinem Vater, Albert Heusch.

${ }^{2}$ Gustav von JORDAN, Louis Jordan. Chronik der Familie Jordan, Berlin 1902, S. 8.

${ }^{3}$ Diese Einschätzung wird von der Forschung bestätigt. Vgl. jüngst Manuela BOHM, Akkulturation und Mehrsprachigkeit am Waisenhaus der Französischen Kolonie in Berlin um 1800, in: Ute TINTEMANN, Jürgen TRABANT (Hg.), Sprache und Sprachen in Berlin um 1800, Hannover 2004, S. 33-54, hier S. 37f.
} 
Pépinière des Chantres et Maîtres d'École - dargelegt werden ${ }^{4}$. $\mathrm{Zu}$ diesem Zweck wird in einem ersten Schritt ein Blick auf Tradition, Struktur und Ausbau des Bildungswesens geworfen, das die Zuwanderer aus Frankreich seit 1685 in Brandenburg-Preußen, insbesondere in Berlin errichtet hatten. Ebenso skizzenhaft wird hiernach erörtert, welche Bedeutung die französische Sprache im Integrationsprozeß erlangte. Sodann rückt das Lehrerseminar in den Mittelpunkt, das seit den 1770er Jahren als eine der ersten Einrichtungen dieser Art Personal für die immer noch zahlreichen französischen Schulen in der Hauptstadt und der Provinz ausbildete. Die Beleuchtung der Gründungsumstände und die Diskussion des Seminarprogramms versprechen die Absichten aufzudecken, welche die Französische Kirche mit der Errichtung des Instituts verband. Abschließend soll eine Rekonstruktion der Absolventenliste Aufschluß darüber geben, welchen Beitrag die Pépinière des Chantres et Maîtres d'École zum Überleben der französischen Gemeinden tatsächlich leisten konnte.

\section{Zum hugenottischen Erziehungswesen}

Bei den Kalvinisten nahm die Kindeserziehung von jeher eine zentrale Stellung ein. In der Tradition des Reformators Johannes Calvin (1509-1564) schrieben sie dem Erziehungsauftrag ihrer Kirche eine hohe Bedeutung $\mathrm{zu}^{5}$. Demgemäß ordnete die $"$ Discipline ecclésiastique des églises reformées de France« an: Les Eglises feront tout devoir de faire dresser des Ecoles, \& donneront ordre que la jeunesse soit instruite ${ }^{6}$. Dieses Gebot führte in Frankreich zur Gründung eines flächendeckenden Netzes an Elementarschulen auf Gemeindeebene, einer Vielzahl von collèges auf Provinzebene und von acht Akademien (dem protestantischen Pendant zu den katholischen Universitäten) auf Landesebene ${ }^{7}$. Geschützt war das protestantische Bildungswesen seit 1598 durch das Edikt von Nantes, das der hugenottischen Minderheit neben anderen Rechten auch die Erziehungsfreiheit im häuslichen und schulischen Bereich

\footnotetext{
${ }^{4}$ Das Amt des Kantors war im 18. Jahrhundert oftmals mit dem Amt des örtlichen Lehrers verbunden, die Ausbildung ähnlich. Hier wird daher verkürzt nur vom Lehrerseminar (anstatt vom Kantoren- und Lehrerseminar) gesprochen.

${ }^{5}$ Zum pädagogischen Programm Calvins vgl. Reinhold HEDTKE, Erziehung durch die Kirche bei Calvin. Der Unterweisungs- und Erziehungsauftrag der Kirche und seine anthropologischen und theologischen Grundlagen, Heidelberg 1969 (Pädagogische Forschungen, Veröffentlichungen des Comenius-Instituts, 39).

${ }^{6}$ [Isaac] D'HUISSEAU (Hg.), La discipline ecclésiastique des eglises reformées de France [etc.], Amsterdam 1710, S. 98.

${ }^{7}$ Zur Kindes- und Jugenderziehung bei den Protestanten in Frankreich vgl. nach wie vor: Paul DE FÉlICE, Les Protestants d'autrefois, Bd. 4: Éducation, instruction: Vie intérieure des églises, mœurs et usages, Paris 1902.
} 
eingeräumt hatte - freilich unter bestimmten Auflagen ${ }^{8}$. Mit dem Regierungsantritt Ludwigs XIV. (1638-1715) im Jahr 1661 verschlechterte sich die Situation seiner protestantischen Untertanen zunehmend. Der innenpolitische Leitsatz des Sonnenkönigs lautete sune foi, une loi, un roik, was unter anderem bedeutete, daß er den Katholizismus zur Staatsreligion machen wollte. Protestanten wurden nun aus immer mehr Berufen und öffentlichen Ämtern ausgeschlossen, die kirchlichen Handlungen überwacht und das reformierte Schulwesen gestört. Die höheren protestantischen Bildungseinrichtungen mußten eine nach der anderen schließen oder gingen an Jesuiten über, die niederen Schulen wurden stark reduziert und ihre Schülerzahl begrenzt. Der siebte Artikel des Edikts von Fontainebleau (1685), mit dem das Edikt von Nantes widerrufen und die inzwischen stark dezimierte Gruppe der Protestanten zur Konversion gezwungen werden sollte, ordnete die Schließung aller reformierten Lehranstalten an?

Das gleiche Edikt untersagte den Hugenotten die Auswanderung aus dem französischen Königreich, nur ihre Pastoren durften emigrieren. Dennoch wagten rund 150000 Menschen die Flucht. Mehrere protestantische Herrscher reagierten sogleich mit Einladungen in der Hoffnung, die als wirtschaftlich und kulturell fortschrittlich geltenden Hugenotten in ihre Länder zu holen. Kurfürst Friedrich Wilhelm I. (1640-1688) gelang es mit seinem im Potsdamer Edikt festgeschriebenen Privilegienprogramm, 15000 dieser Emigranten für eine dauerhafte Ansiedlung in Brandenburg-Preußen zu gewinnen. Bevorzugter Ansiedlungsort wurde Berlin, wo sich bis 1700 etwa 6000 dieser Flüchtlinge niederließen. Jeder vierte Berliner war um die Wende zum 18. Jahrhundert somit französischer Abstammung ${ }^{10}$.

Unsicherheiten hinsichtlich der Gültigkeit der kalvinischen Kirchenordnung in seinen Territorien räumte Kurfürst Friedrich III. (1688-1713, seit 1701 als

\footnotetext{
${ }^{8}$ Das Edikt von Nantes findet sich abgedruckt in: Élie BENOIST, Histoire de l'Edit de Nantes, contenant Les choses les plus remarquables qui se sont passées en France avant \& après sa publication [etc.], Bd. 1, Delft 1693, S. 63-98; Athanase-Jean-Léger JOURDAN u.a. (Hg.), Recueil général des anciennes lois françaises, depuis l'an 420 jusqu'à la Révolution de 1789 [etc.], 29 Bde., Paris 1821-1833, hier Bd. 15: août 1589-mai 1610, hg. von François-André ISAMBERT, Alphonse-Honoré TAILLANDIER, DECRUSY, Nr. 124f., S. 170-210. Die wohl signifikanteste Auflage war, daß von den Reformierten nur an solchen Orten Schulen unterhalten werden durften, wo ihnen auch die öffentliche Kultusausübung gestattet war.

${ }^{9}$ Edikt von Fontainebleau, abgedruckt bei Peter LIESSEM, Die Aufhebung des Edikts von Nantes (1598) durch das Edikt von Fontainebleau (1685), Sickte 1987 (Geschichtsblätter des Deutschen Hugenotten-Vereins, 19/8), S. 10; JouRDAN u.a. (Hg.), Recueil général (wie Anm. 8), hier Bd. 19: janvier 1672-mai 1686, hg. von François-André ISAMBERT, AlphonseHonoré TAILLANDIER, DECRUSY, Nr. 1192, S. 530-534.

${ }^{10}$ Die Zahlen beruhen auf einer Auswertung der Kolonielisten durch François DAvid, Les colonies des réfugiés protestants français en Brandebourg-Prusse (1685-1809): institutions, géographie et évolution de leur peuplement, in: Bulletin de la Société de l'histoire du protestantisme français 140 (1994), S. 111-141, hier S. 124f. und S. 130.
} 
Friedrich I. König in Preußen) 1689 aus, indem er den jungen französischen Kirchen die Beachtung ihrer "Discipline ecclésiastique« vorschrieb ${ }^{11}$. Diese Anordnung stellte die rechtliche Grundlage für das eigenständige Bildungswesen der Berliner Hugenotten dar. Keine Übernahme fand indes das synodale Element der »Discipline ecclésiastique«. Der Kurfürst bestand auf seine Episkopalrechte und bekräftigte diese Haltung durch die Einsetzung der Commission ecclésiastique (1694), aus der im Juli 1701 das Französische Oberkonsistorium hervorging ${ }^{12}$. Dieses stand fortan als oberste geistliche Behörde mit administrativen und justiziellen Funktionen den französischen Kolonien vor. Für den Erziehungssektor war es insofern maßgebend, als es bis zu seiner Auflösung im Dezember 1808 die oberste Entscheidungskompetenz in Angelegenheiten des französisch-reformierten Schulwesens innehatte ${ }^{13}$.

Für Organisation und Ordnung des Schullebens in den einzelnen Stadt- und Landgemeinden zeichnete das jeweilige Konsistorium verantwortlich. Das größte Gremium der Französischen Kirche in Berlin, die Assemblée générale oder auch Compagnie du Consistoire, war mit Schulangelegenheiten nur am Rande betraut. Ihm gehörten die Prediger, Ältesten und Diakone der verschiedenen Parochien an, in besonderen Fällen um zehn bis dreißig Familienoberhäupter verstärkt. Bei den im vierzehntägigen Turnus stattfindenden Sitzungen wurde über all die Angelegenheiten entschieden, die nicht in den Kompetenzbereich von Konsistorium oder Diakonat fielen, unter anderem über die Ernennung von Kirchenbeamten und Lehrern sowie die Aufnahme von Zöglingen in das theologische oder in das Lehrerseminar. Ferner durften neue Reglements, also auch die für das Schulwesen relevanten, grundsätzlich nur mit Zustimmung der Assemblée générale eingeführt werden ${ }^{14}$. Die Angelegenheiten der Jugendpflege fielen in den Aufgabenbereich des Mittwochs-Konsistoriums, zur besseren Unterscheidung von den beiden anderen Konsistorien nach dem Tag seiner Zusammenkünfte so genannt. Es übte das Kirchenregiment aus und hatte somit auch die Erfüllung des kirchlichen Erziehungsauftrages sicherzustellen. Entsprechend formulierten die Gemeindesatzungen: Das Mittwochs-

${ }^{11}$ Christian Otto MYLIUS, Recueil des Edits, Ordonnances, Reglements, et Rescripts, contenant les Privileges et les Droits attribues aux François Refugiés [etc.], Berlin 1750, Nr. 20 (07.12.1689), Sp. 71-74.

${ }^{12}$ Vgl. hierzu Ernst MENGIN, Das Recht der französisch-reformierten Kirche in Preußen. Ein Urkundenbuch, Berlin 1929, S. 4-8; und jüngst Eckart BIRNSTIEL, Le statut juridique des églises huguenotes en Brandebourg-Prusse (1672-1809), in: Hubert BOST, Claude LAURIOL (Hg.), Refuge et désert. L'évolution théologique des huguenots de la Révocation à la Révolution française, Paris 2003, S. 33-53, hier S. 38-47.

${ }^{13}$ Die Verordnungen und Zirkular-Verfügungen zum öffentlichen Unterricht (erhalten für die Jahre 1743-1786) finden sich im Geheimen Staatsarchiv Preußischer Kulturbesitz (= GStA PK) I. HA Rep. 76 alt (Ältere Oberbehörden für Wissenschaft, Kunst, Kirchen- und Schulsachen), VI, Nr. 25.

${ }^{14}$ Reglements für die Compagnie des Consistoriums der französischen Kirche zu Berlin, hg. v. der Compagnie des Consistoriums, Berlin 1876, Abschn. 1, Kap. 1,3 und Kap. 2,9-11. 
Konsistorium hat »über Alles, was den Unterricht und die Erziehung der Jugend betriff, zu wachen « und "von dem Zustande der Schulen Kenntnis zu nehmen ${ }^{15}$. Die Aufsicht, die es bis ins 19. Jahrhundert über das französische Schulwesen ausübte, erstreckte sich auf die Bereiche Genehmigung und Visitation der Schulen, Überprüfung der Schulmeister auf fachlichem und sittlichem Gebiet sowie Kontrolle des regelmäßigen Schulbesuchs der Kinder ${ }^{16}$. Unterstützt wurde das Konsistorium in seiner Arbeit von einer eigens hierfür eingesetzten Schulkommission.

So wie die französische Schulverwaltung über Jahrzehnte gewachsen war, war auch das gemeindliche Erziehungs- und Bildungswesen gediehen. In den Protokollen des Konsistoriums ist erstmals im Juni 1686 von einem Gemeindelehrer die Rede, der einige Armenkinder im Lesen unterrichten sollte ${ }^{17}$. Knapp sechs Jahre später wurde die erste ordentliche Gemeindeschule mit schon erweitertem Curriculum gegründet ${ }^{18}$. Was so klein begonnen hatte, präsentierte sich beim hundertsten Jahrestag des Edikts von Potsdam als differenziertes und effizientes Bildungswesen. Zu diesem Zeitpunkt verfügte die französische Gemeinde Berlins über mehr als 60 Elementarschulen, zahlreiche Pensionate, ein Waisenhaus, ein Kinderhospital, mehrere private höhere Schulen, ein Gymnasium und jeweils ein Seminar für Lehrer und Theologen ${ }^{19}$. Mit diesen Einrichtungen leistete die Kirche ihrem Erziehungsauftrag gewissenhaft Folge. Neben diesen Erziehungsauftrag aber trat seit der zweiten Hälfte des 18. Jahrhunderts - als die Nachfahren der Refugiés ihrer Französischkompetenz verlustig gingen, als immer weniger von ihnen am Abendmahl teilnahmen ${ }^{20}$,

${ }^{15}$ Ibid., Abschn. 1, Kap. 3,3 und 3,4.

${ }^{16}$ Zusammengefaßt sind die Zuständigkeiten und Privilegien des Konsistoriums in einem Bericht, den man 1809 auf Anfrage Wilhelm von Humboldts (1767-1835) verfaBte. Vgl. Archiv der Französischen Kirche im Französischen Dom, Berlin (= AFrD) Rep. 04-660, Bl. 180. Humboldt war 1809 Leiter der Sektion für Kultus und Unterricht im preuBischen Innenministerium geworden.

${ }^{17}$ Ibid., Rep. 04-2400, S. 42 (02.03.1687) und S. 44 (27.03.1687).

${ }^{18}$ GStA PK (wie Anm. 13), I. HA Rep. 122 (Französisches Koloniedepartement), 3 b II, Bd. 1, Bl. 22 (17.02.1792). Zum Lehrprogramm gehörten nun Lesen, Schreiben und Rechnen.

${ }^{19}$ Einen summarischen Überblick über das hugenottische Schulwesen in Berlin bietet Eckart BIRNSTIEL, Zwischen zwei Kulturen. Die Schule der Berliner Hugenotten, in: Mitteilungen und Materialien der Arbeitsgruppe Pädagogisches Museum e.V. 25 (1987), S. 99-135. Vgl. demnächst ausführlich Franziska ROOSEN, „Soutenir notre Église“. Hugenottische Erziehungskonzepte und Bildungseinrichtungen im Berlin des 18. Jahrhunderts, Bad Karlshafen [2007] (Geschichtsblätter der Deutschen Hugenotten-Gesellschaft e.V., 42).

${ }^{20}$ Vgl. beispielsweise François DAVID, Les colonies françaises en Brandebourg-Prusse: Une étude statistique de leur population, in: Manuela BOHM, Jens HASELER, Robert VIOLET (Hg.), Hugenotten zwischen Migration und Integration. Neue Forschungen zum Refuge in Berlin und Brandenburg, Berlin 2005, S. 69-93, hier S. 85-91. 
als Hochzeiten zwischen Franzosen und Deutschen gang und gäbe wurden ${ }^{21}$ ein weiteres, als mindestens ebenso wichtig erachtetes Anliegen: die schwindende Gruppenidentität der Hugenotten über die Sozialisation des Nachwuchses neu zu prägen und zu erhalten.

\section{Bewahrung der französischen Sprache}

»Eine kollektive Identität begründet sich zum einen auf ein Bewußtsein der Gruppe von sich selbst, zum anderen als Urteil und Zuschreibung >von außen<, d.h. seitens anderer Gruppen; kollektive Identität und Zugehörigkeitsdefinitionen werden über Grenzziehungen der ethnischen Gruppen selbst wie über Abgrenzungen durch andere ethnische Kollektive bestimmt«, schreibt der Soziologe Friedrich Heckmann ${ }^{22}$. Welche Bedeutung der Sprache in diesem von der Kirchenleitung gewollten Abgrenzungsprozeß zukam, läßt sich an der Sprachpolitik der französischen Konsistorien beobachten.

Der französisch-deutsche Sprachwechsel hatte mehrere Etappen durchlaufen, von einer französischen Einsprachigkeit über eine französisch-deutsche Mehrsprachigkeit bis hin zu einer deutschen Einsprachigkeit ${ }^{23}$. Je nach Lage und Größe der Kolonien sowie sozialem Kontext der einzelnen Familien hatte dieser Prozeß früher oder später eingesetzt. Anhand der Sitzungsprotokolle des Berliner Konsistoriums läßt sich nachweisen, daß spätestens ab den 1760 er Jahren eine wachsende Zahl von Gemeindegliedern dem Gottesdienst in französischer Sprache nicht mehr folgen konnte. Die Kirchenleitung aber bestand weiterhin auf der hergebrachten Kultussprache, selbst wenn sie damit den Erfolg ihres seelsorgerischen Auftrags gefährdete. Sie wähnte, daß mit Aufgabe ihrer sprachlichen Eigenständigkeit eine Vereinigung mit den deutschreformierten Kirchen drohen und sie damit ihre Autonomie verlieren würde ${ }^{24}$. Insbesondere die Wahrung der französischen Sprache versprach, die Distinktion von den Einheimischen offensichtlich zu machen.

${ }^{21}$ Vgl. beispielsweise Susanne LACHENICHT, Migration, Migrationspolitik und Integration. Hugenotten in Brandenburg-Preußen, Irland und Großbritannien, in: ibid., S. 37-58, hier S. 50-53.

${ }_{22}$ Friedrich HECKMANN, Ethnische Minderheiten, Volk und Nation: Soziologie inter-ethnischer Beziehungen, Stuttgart 1992, S. 55.

${ }^{23}$ Hierzu jüngst: Manuela BOHM, Der französisch-deutsche Sprachwechsel in der hugenottischen Landkolonie Strasburg/Uckermark, in: BOHM, HÄSELER, VIOLET (Hg.), Hugenotten (wie Anm. 20), S. 135-153. Vgl, auch ihren Beitrag im vorliegenden Band.

${ }^{24}$ Vgl. Frédéric HARTWEG, Sprachwechsel und Sprachpolitik der Französisch-Reformierten Kirche in Berlin im 18. Jahrhundert, in: Jahrbuch für die Geschichte Mittel- und Ostdeutschlands 30 (1981), S. 162-176. 
Nach außen wurde die französische Sprache somit zu einem existenzlegitimierenden Moment. Als markanter Ausdruck des Andersseins der Hugenotten sollte sie deren privilegierte Sonderexistenz rechtfertigen. Nicht zu Unrecht galt den Führungsgremien der Hugenotten das Französische als Symbol der Eigenständigkeit, kann doch allgemein beobachtet werden, daß sich ihre Gemeinden erst dann auflösten, wenn ihre französischsprechenden Mitglieder ausstarben $^{25}$. Nach innen wurde die Beibehaltung der Sprache der Vorväter zu einer Strategie der Identitätssicherung. Das Französische wurde zu einem "monument de mémoire collective «" ${ }^{26}$. Uber die Sprache sollte ein Wir-Gefühl transportiert werden, das dem Zusammenhalt der Gemeinde nur nützen konnte, indem es an die gemeinsamen Wurzeln und die Exklusivität der hieraus hervorgegangenen Gemeinschaft erinnerte. Im Kampf um die Aufrechterhaltung des Koloniestatus entwickelte sich die französische Sprache somit zu einem Prestigeobjekt der Kirchenleitung.

In diesem Kontext gelangten die Schulen zu einer ganz neuen Bedeutung. Hier konnte man während entscheidender Jahre prägenden Einfluß auf eine Vielzahl der Koloniekinder ausüben, hier konnte man ihnen beibringen, was in ihrem Elternhaus vielleicht schon vergessen war. Wo ließ sich Sprachpflege intensiver betreiben als im Klassenzimmer? Wo konnte man besser die Besonderheiten des eigenen Bekenntnisses vermitteln? Wo sollte die gemeinsame Herkunft offensichtlicher werden als in einer Gruppe hugenottischer Kinder? $\mathrm{Daß}$ dem Französischen Oberkonsistorium solche Gedankengänge nicht fern lagen und ihm die Schule durchaus als Mittel zur Bewahrung und Festigung kultureller Eigenständigkeit erschien, zeigen diverse Anordnungen aus jenem Zeitraum. Von den beiden, die hier genannt werden sollen, sei zunächst das Reglement angefuihrt, das die Schulkommission im Auftrag der Compagnie du Consistoire im September des Jahres 1773 für die französischen Gemeindeund Kolonieschulen ausarbeitete ${ }^{27}$.

Man sei darauf hingewiesen worden, heißt es dort einleitend, daß die Verwendung der Muttersprache an den Schulen dermaßen nachgelassen habe, daß hier nicht nur die Kinder der Deutschen kaum noch die französische Sprache erlernen könnten, sondern selbst die Kinder der Franzosen ihre geringen Fran-

${ }^{25}$ Jürgen EschmaNN, Die Sprache der Hugenotten, in: DERS. (Hg.), Hugenottenkultur in Deutschland, Tübingen 1988 (Erlanger Romanistische Dokumente und Arbeiten, 2), S. 9-35, hier S. 12.

${ }^{26}$ Myriam YARDENI, Le Refuge allemand et la France. Histoire d'une aliénation, in: Frédéric HARTWEG, Stefi JERSCH-WENZEL (Hg.), Die Hugenotten und das Refuge. Deutschland und Europa, Berlin 1990 (Einzelveröffentlichungen der Historischen Kommission zu Berlin, 74), S. 187-203, hier S. 187.

${ }^{27}$ AFrD (wie Anm. 16), Rep. 04-660, Bl. 52 (08.09.1773). 
zösischkenntnisse vergäßen ${ }^{28}$. Dies rühre vor allem daher, daß die Lehrer den Schülern erlaubten, sich untereinander auf Deutsch zu unterhalten und selbst häufiger mit ihnen deutsch als französisch sprächen.

Mit folgenden Maßnahmen gedachte die Schulkommission, den Gebrauch der französischen Sprache zu fördern: Französisch sollte fortan in der Schulstube Unterrichts- und Umgangssprache sein. Des Deutschen durfte sich der Lehrer nur bedienen, wenn er den Kindern einen Sachverhalt erklären mußte, den diese bei französischer Unterrichtung zuvor nicht begriffen hatten. Ferner hatte der Lehrer darauf zu achten, von den Kindern ausschließlich in französischer Sprache angeredet zu werden - nur in Ausnahmefällen und mit seiner speziellen Erlaubnis sollten sie ihre Fragen in deutscher Sprache formulieren dürfen. Auch untereinander durften die Schüler nur französisch sprechen (eigentlich sollten sie sich gar nicht miteinander unterhalten). Zwei Unterrichtsstunden mußten fortan pro Woche der französischen Sprache vorbehalten sein: In dieser Zeit sollten Vokabeln, Gesprächssituationen und Übersetzungen vom Deutschen ins Französische und umgekehrt geübt werden. Da die meisten Kinder sich das empfohlene Lehrbuch nicht leisten konnten (es handelte sich um die französisch-deutsche "Grammatik» von Robert Jean des Pepliers, der Gespräche, Redensarten, Denksprüche etc. in beiden Sprachen angehängt waren), wollte die Compagnie du Consistoire jedem Lehrer eine Ausgabe überreichen. In ihrem Reglement präzisierte die Schulkommission sogar die Methode, derer der Lehrer sich bei den Sprachübungen zu bedienen hatte: Zuerst sollte er ein Wort auf Französisch und auf Deutsch laut vorlesen, und es anschließend jeden Schüler wiederholen lassen; dann folgten Sätze. Am Ende eines Dialogs oder eines Kapitels fand eine Repetition statt, bei der jeder Schüler auf eine bestimmte Frage des Lehrers zu antworten hatte. Außerdem sollten mündlich die Fragen und Antworten des »Kleinen Katechismus « von Superville - auch dieses Buch wurde den Lehrem an die Hand gegeben - vom Deutschen ins Französische und zurück übersetzt werden.

Ob die vom Konsistorium bestimmten Maßnahmen von Erfolg gekrönt waren, geht aus dem Quellenmaterial nicht unmittelbar hervor. Die Schulkommission zumindest wurde angehalten, auf die Umsetzung des Reglements zu achten ${ }^{29}$. So hatte man bereits einige Monate vor seiner Publikation der Witwe Aumeras die Erlaubnis zur Gründung einer Schule mit der Begründung verweigert, daß sie die französische Sprache nicht beherrsche ${ }^{30}$.

Die zweite Anordnung datiert vom März 1769. Es entbehrt nicht einer gewissen Ironie, daß sich just die oben erwähnte Strategie - am Französischen

${ }^{28}$ Tatsächlich besuchten viele deutsche Kinder die französischen Schulen. Vor allem der zweisprachige Unterricht und das oftmals moderate Schulgeld scheinen ihre hohe Anziehungskraft ausgemacht zu haben.

${ }^{29}$ AFrD (wie Anm. 16), Rep. 04-660, B1. 52 (08.09.1773).

${ }^{30}$ Ibid., Rep. 04-241 1, S. 191 (14.07.1773). 
als Liturgiesprache festzuhalten - als ursächlich für diese Anordnung erweist. Die durch diese Sprachpolitik hervorgerufenen Verständnisprobleme nämlich sorgten für immer mehr Übertritte zur deutsch-reformierten Kirche. Die Betroffenen argumentierten, sich in ihren eigenen Kirchen nicht mehr erbauen, geschweige denn dem Gottesdienst folgen zu können. Alarmiert vom Mitgliederschwund in den eigenen Reihen, wurde das Französische Oberkonsistorium endlich tätig. Die Art und Weise, wie man den Austritten entgegenzusteuern beschloß, mutet freilich etwas befremdlich an. Abhilfe nämlich sollte nicht etwa ein Wechsel der Kultussprache schaffen, sondern die einzelnen Schulmeister. Ihnen wurde aufgetragen, für die Bewahrung der französischen Sprache unter ihren Schülern Sorge zu tragen:

Chers \& bien aimés salut! Plusieurs membres des Églises françaises se déterminant depuis quelque tems à demander des Lettres Dimissoires [sic] sous pretexte de ne pas entendre suffisamment la langue française, ce qui tend au détriment de Nos Colonies, Nous vous enjoignons par les présentes, 1. d'ordonner le plus sérieusement de Nôtre part à vos Maîtres d'Ecole de s'acquitter fidellement de leur devoir à cet égard en donnant aux enfans qu'ils instruisent toutes les instructions \& tous les secours possibles pour leur rendre la langue française familiere; \& 2. de déclarer à tous les membres de vôtre troupeau que ceux qui négligeront d'instruire leurs enfans dans ladite langue, \& qui sans des raisons suffisantes quitteront l'Eglise française seront privés de tous les avantages dont eux \& leurs enfans auroient pu jouir, \& qu'ils auroient pu obtenir de Nos établissemens \& fondations pieuses ${ }^{31}$.

Da zahlreiche Eltern des Französischen nicht mehr mächtig waren, erschien die Idee sinnvoll, den Lehrern die Spracherziehung zu übertragen - wären da nicht zwei signifikante Schwierigkeiten aufgetreten: Zum einen war es um die Versorgung der französischen Schulen in jenen Jahren schlecht bestellt, viele mußten aufgrund Lehrermangels schließen ${ }^{32}$. Zum anderen galt das vorhandene Lehrpersonal vielerorts als nicht ausreichend qualifiziert. Die Berliner Ecole de Charité als größte unter allen französischen Elementarschulen in Brandenburg-Preußen etwa erklärte selbst, oftmals gezwungen gewesen zu sein, höchst mittelmäßige Lehrer anzustellen, weil bessere nicht hatten gefunden werden können ${ }^{33}$.

${ }^{31}$ GStA PK (wie Anm. 13), I. HA Rep. 76 alt (Ältere Oberbehörden für Wissenschaft, Kunst, Kirchen- und Schulsachen), VI, Nr. 25, Bl. 117 (25.03.1769).

${ }^{32} \mathrm{Vgl}$. für Magdeburg, Halberstadt und Burg: Henri TOLLIN, Geschichte der Franzősischen Colonie von Magdeburg, 6 Bde., Halle 1886-1894, Bd. 3/1C, S. 808; AFrD (wie Anm. 16), Rep. 04-1523, Bl. 117 (22.02.1779).

${ }^{33}$ Bibliothek des Französischen Doms, Berlin (= BFrD), L-111 (Relation de l'École de Charité, 1775), S. 5. Für erste Informationen zur Ecole de Charité vgl. Eduard MURET, Geschichte der französischen Kolonie in Brandenburg-Preußen, unter besonderer Berücksichtigung der Berliner Gemeinde, Berlin 1885, S. 157-166. 


\section{Die Pépinière des Chantres et Maîtres d'École}

Bereits wenige Monate nach der Verfügung vom März 1769 mußte das Oberkonsistorium daher erneut handeln. Ihm war bekannt, daß die École de Charité seit geraumer Zeit ehemalige Schüler zu Lehrern weiterbildete. In einer geregelten Lehrerausbildung offenbar die Lösung für seine drängenden Probleme sehend, ermunterte es die Schulleitung, dieses Projekt auszubauen. Die Direktion der Ecole de Charité konnte aber damals nur die Ausbildung einiger weniger Jungen zusagen, weil es ihr an Räumlichkeiten mangelte ${ }^{34}$. Als fünf Jahre später der Neubau an der Jägerstraße fertiggestellt war, sollte die Lehrerausbildung professioneller aufgezogen werden ${ }^{35}$. In der Zwischenzeit hatte sich der Bedarf an geeigneten Lehrkräften immer stärker abgezeichnet, und sowohl die Armenschule als auch mehrere französische Kolonien litten unter der schlechten Versorgungslage. Auch hatte das Oberkonsistorium erneut interveniert. 1775 aber konnten die Leiter der École de Charité in ihrem Jahresbericht endlich vermelden, vier ständige Plätze für die Unterweisung künftiger Lehrer geschaffen zu haben. Unter der Aufsicht eines Lehrers sollten diese Schüler Anleitungen erhalten, die sie für ihren späteren Beruf qualifizierten: La lecture \& l'écriture françoise \& allemande, l'orthographie, l'arithmétique, le chant, une instruction plus détaillée, \& plus approfondie dans la Religion sont les objets dont ils seront uniquement occupés ${ }^{36}$. Waren die jungen Männer in ihren Studien ausreichend fortgeschritten und hatten ein bestimmtes, nicht weiter präzisiertes Alter erreicht, sollten sie sich an der Ecole de Charité üben können. Wenn sie sich durch Leistungen und Betragen bewährt hatten, sollten Anstellungen für sie gesucht werden. Erster Kandidat des Programms an der Armenschule war ein gewisser Daniel Nicolas ${ }^{37}$. Bereits nach zwei Jahren konnte dieser auf Anfrage der dortigen Kirche nach Halberstadt gehen, wo er zunächst Hilfslehrer der Kolonieschule wurde, um kurz darauf Nachfolger des Hauptlehrers zu werden ${ }^{38} .1788$ kehrte er nach Berlin zurück. Hier wurde er anfangs Lehrer an der Domschule, dann Aufseher an der Pépinière ${ }^{39}$. Ein zweiter Kandidat dieses Programms trat 1777 eine Stelle in Frankfurt/Oder $\mathrm{an}^{40}$.

${ }^{34}$ AFrD (wie Anm. 16), Rep. 04-817, S. 321 (11.09.1769).

${ }^{35}$ BFrD (wie Anm. 33), L-111 (Relation de l'École de Charité, 1775), S. 4; AFrD (wie Anm. 16), Rep. 04-818, S. 406 (13.02.1775).

${ }^{36}$ BFrD (wie Anm. 33), L-111 (Relation de l'École de Charité, 1775), S. 5.

${ }^{37}$ Ibid., S. 6.

${ }^{38}$ Ibid., L-111 (Relation de l'École de Charité, 1777), S. 6.

${ }^{39}$ Vgl. ibid., L-111 (Relation de l'École de Charité, 1790), S. 4; AFrD (wie Anm. 16), Rep. 04-504, Nr. 309. Einige Jahre später machte er sich mit einem Mädchenpensionat selbständig.

${ }^{40}$ BFrD (wie Anm. 33), L-11 (Relation de l'École de Charité, 1777), S. 6. 
Von derartigen Erfolgen überzeugt, beschloß die Compagnie du Consistoire schließlich in enger Zusammenarbeit mit der Leitung der École de Charité die Gründung einer eigenständigen Institution. Eine Kommission arbeitete hierfür ein Konzept aus, das dem Oberkonsistorium, der Versammlung der Familienväter und den Kirchen des Landes vorgelegt wurde ${ }^{41}$. Nach deren Genehmigung vereinbarte die Compagnie du Consistoire mit der École de Charité, daß die Pépinière zwar unabhängig von dieser bestehen, die Kandidaten aber zunächst gegen eine fixe Pension von 25 Reichstalern in deren Räumlichkeiten untergebracht werden sollten. Finanziert und geleitet wurde das neue Institut von der Compagnie du Consistoire ${ }^{42}$. Am 20. Januar 1779 wurde das von der Kommission vorgelegte Generalreglement gebilligt, und am 5. Oktober 1779 konnte das Lehrerseminar eröffnet werden ${ }^{43}$.

Damit besaßen die Hugenotten eines der wenigen Lehrerseminare jener Zeit. Tatsächlich stellte die Lehrtätigkeit bis ins 19. Jahrhundert hinein keinen eigenständigen, hauptamtlichen Beruf dar: Küster, Handwerker, Schneider, Kesselflicker und (invalide) Soldaten, zuweilen auch Gymnasiasten betätigten sich gleichermaßen auf dem Gebiet der institutionellen Kindererziehung. Hatte der Lehrer einer niederen Schule überhaupt eine Ausbildung erhalten, so durch Anleitung eines älteren Kollegen oder eines Geistlichen ${ }^{44}$. Daneben gab es immer wieder Schulmeisterdynastien, in denen die Väter ihre Söhne anleiteten, bevor diese ihnen im Amt nachfolgten ${ }^{45}$. Bis zur Wende zum 19. Jahrhundert zählt Michael Sauer rund zehn Seminargründungen für die Ausbildung preußischer Elementarschullehrer ${ }^{46}$.

Aufgenommen in die Pépinière des Chantres et Maîtres d'École wurden hugenottische Jugendliche, die das Alter von 14 Jahren erreicht haben mußten. Ihr Lehrplan, der im Generalreglement fixiert ist, führt folgende Unterrichtseinheiten auf:

1. la lecture, et l'écriture française, et Allemande, qu'il faut tacher de porter à la plus grande perfection possible, en leur donnant une idée Elémentaire de la Grammaire françoise.

2. l'Arithmétique.

3. le Chant des Psaumes [...]

${ }^{41}$ Ibid., L-111 (Relation de l'École de Charité, 1778), S. 5.

${ }^{42}$ AFrD (wie Anm. 16), Rep. 04-819, S. 127 (14.12.1778), S. 160 (11.10.1779).

${ }^{43}$ Festgehalten ibid., Rep. 04-2413, S. 17-21 (27.01.1779). Eine leicht modifizierte Fassung der Anstaltssatzungen findet sich in: Reglements der Compagnie (wie Anm. 14), Abschn. 1, Kap. 36.

44 Michael SAUER, Volksschullehrerbildung in Preußen. Die Seminare und Präparandenanstalten vom 18. Jahrhundert bis zur Weimarer Republik, Köln, Wien 1987 (Studien und Dokumentationen zur deutschen Bildungsgeschichte, 37), S. 11.

45 Wolfgang NEUGEBAUER, Absolutistischer Staat und Schulwirklichkeit in BrandenburgPreußen. Mit einer Einführung von Otto Büsch, Berlin, New York 1985 (Veröffentlichungen der Historischen Kommission zu Berlin, 62), S. 368-370.

${ }^{46}$ SAUER, Volksschullehrerbildung (wie Anm. 44), S. 15. 
4. la Religion dont il importe de les instruire, de manière qu'ils soient en état, d'en donner aux enfans la coñoissance élémentaire.

5. une connoissance élémentaire de l'histoire de la Géographie, ainsi que quelques notions de l'histoire naturelle $e^{47}$.

Bei einem Vergleich mit den Curricula anderer zeitgenössischer Lehrerseminare fällt auf, daß sich die Lehrpläne trotz der verschiedenen Anstaltsträger kaum unterschieden - bis auf zwei Ausnahmen: Zum einen wurde an der Pépinière des Chantres et Maîtres d'École besonderes Augenmerk auf die Vermittlung der spezifischen Glaubensinhalte gelegt. Dazu dienten neben den Unterrichtsfächern Psalmensingen und Religion die täglichen Frömmigkeitsübungen im Seminar (Gebete, Bibellektüre und eben Psalmensingen), der Besuch der sonntäglichen Gottesdienste in der friedrichstädtischen Kirche und die Niederschrift der dort gehörten Predigten. Zum anderen bildete der Unterricht im Französischen, das selbstverständlich auch Umgangssprache war, einen Schwerpunkt der Ausbildung. Daß die Hugenotten ein eigenes Lehrerseminar eingerichtet hatten und unter erheblichen (finanziellen) Anstrengungen bis 1836 am Leben halten konnten ${ }^{48}$, läßt sich also weniger auf ein besonderes Wissensprogramm zurückführen. Seine Gründung erklärt sich vielmehr aus der Absicht, die französischen Erziehungsinstitute in Stadt und Land mit einem adäquat geschulten Personal zu versorgen, das heißt: mit Lehrern, die in Glaubensfragen firm und in der französischen Sprache geübt waren.

Tatsächlich gelang es den hugenottischen Führungsinstanzen mit Hilfe des Seminars, die französische Schullandschaft in Brandenburg-Preußen wiederzubeleben. Die Reichweite des Seminars war bemerkenswert. Ein Jahr nach seiner Gründung tauchen in den Schülerlisten, die auf den letzten Seiten der Jahresberichte der École de Charité abgedruckt wurden, erstmals auch die Zöglinge der Pépinière auf. 1780 zählte sie sieben Schüler, davon vier Neuzugänge $^{49} .1782$ waren es acht Schüler, und in den nächsten Jahren schwankte ihre Zahl kontinuierlich zwischen sieben und neun ${ }^{50}$. Seit 1784 wurden dem Leser der Relationen auch Art und Ort der Anstellung mitgeteilt, die die Absolventen der Pépinière inzwischen gefunden hatten. Aufgeführt wurden in diesem Jahr sechs Jungen: Philippe Cordier hatte eine Anstellung als Küster und Schulmeister der französischen Kirche in Schwedt gefunden, Jean Betac

${ }^{47}$ AFrD (wie Anm. 16), Rep. 04-2413, S. 17-21 (27.01.1779), Art. 15.

${ }^{48}$ Zum ersten Mal hatte der Betrieb der Pépinière zwischen 1809 und 1817 eingestellt werden müssen. Auch die Schließung 1836 war nicht endgültig: Von 1855 bis 1872 bestand das Lehrerseminar als Konvikt, und noch bis 1908 konnte aus seinem ehemaligen Fonds die Ausbildung von Seminaristen am Lehrerseminar in Köpenick finanziert werden. Vgl. Ursula FUHRICH-GRUBERT, Die Französische Kirche zu Berlin. Ihre Einrichtungen 1672-1945, Bad Karlshafen 1992 (Tagungsschriften des Deutschen Hugenotten-Vereins, 11), S. 39.

${ }^{49}$ BFrD (wie Anm. 33), L-111 (Relation de l'École de Charité, 1780), S. 14.

${ }^{50}$ Eine Ausnahme bildet das Jahr 1788 , in dem nur fünf Lehramtskandidaten die Pépinière besuchten. 
als Lehrer am Magdeburger französischen Waisenhaus, Jean Malbran als Lehrer am Magdeburger wallonischen Waisenhaus, Pierre Sarre als Küster und Schulmeister in Strasburg/Uckermark, und Jean Cazalét war nach Schlesien gegangen $^{51}$. Der sechste Absolvent, Jean George, unterrichtete als Schreiblehrer an der École de Charité.

Zwar erlaubt die lückenhafte Überlieferung keine systematische Analyse der späteren Karrieren der Absolventen, doch läßt die folgende Übersicht einige allgemeine Tendenzen erkennen: Nach einer durchschnittlichen Studiendauer von vier Jahren schlug ein Großteil der Schulabgänger eine pädagogische Laufbahn ein. Dabei fällt das breite geographische Tätigkeitsfeld ehemaliger Schüler der Pépinière auf. Während nur fünf der insgesamt dreißig faßbar werdenden Absolventen als Buchstabierlehrer an der École de Charité oder als Aufseher an der Pépinière verblieben und vier von ihnen in Berlin Karriere machten, fand ein Großteil in Instituten Anstellung, die sich über die gesamte Mark Brandenburg verteilten. Die Beispiele Pierre Sy und Jean Jacob Noé, die als Lehrer in Königsberg bzw. in Wesel tätig waren, verweisen darauf, daß Absolventen der Pépinière sogar außerhalb dieses engeren Tätigkeitsgebietes gefragt waren ${ }^{52}$.

Absolventen der Pépinière mit anschließender Anstellung (1774-1815)

\begin{tabular}{|l|l|c|l|}
\hline 1. Nennung & \multicolumn{1}{|c|}{ Schüler } & Austrittsjahr & \multicolumn{1}{|c|}{ Anstellung/Fortbildung } \\
\hline & Jean Maire & 1774 & $\begin{array}{l}\text { Buchstabierlehrer und Kommis- } \\
\text { sionär der École de Charité }\end{array}$ \\
\hline & David Guiard & 1779 & $\begin{array}{l}\text { Buchstabierlehrer } \\
\text { der École de Charité }\end{array}$ \\
\hline
\end{tabular}

${ }^{51}$ BFrD (wie Anm. 33), L-111 (Relation de l'École de Charité, 1784), S. 13.

52 Die Daten der folgenden Tabelle stammen von mir ergänzt aus: Ibid., L-111 (Relationen 1765-1815). Verzeichnet wurden hier nur die Absolventen der Pépinière, zu deren späterer Anstellung Informationen überkommen sind. Erläuterung zur Tabelle: $1 / J a h r$ der Ersterwähnung: Angegeben wird hier, wann ein Schüler erstmals in den Zöglingslisten auftaucht, die am Ende der jährlich publizierten Relationen der École de Charité abgedruckt wurden. 2/Schüler: Ist der Name mit einem (*) versehen, bedeutet dies, daß der Schüler in diesem Jahr in die Pépinière aufgenommen wurde. Bei den übrigen Personen handelt es sich zwar um eine Ersterwähnung, doch muß dies nicht unbedingt heißen, daß es sich zugleich um ihr erstes Ausbildungsjahr handelt. 3/Austrittsjahr: Angegeben wird hier, wann der Schüler die Pépinière nachweislich verlassen hat. 4/Anstellung: Bei vielen Absolventen der Pépinière wurde vermerkt, welche Art von Anstellung sie wo gefunden hatten. Bei anderen indes beschränkte man sich auf die Bemerkung, daB sie eine Anstellung gefunden hatten, oder auf eine Notiz, welchen Weg sie einzuschlagen gedachten. 


\begin{tabular}{|c|c|c|c|}
\hline 1782 & $\begin{array}{l}\text { Jean Malbran } \\
\text { Jean Godefroy Patet } \\
\text { Pierre Cordier } \\
\text { *Pierre Sy } \\
\text { Benjamin Prestiot } \\
\text { Jean George } \\
\text { Jean Betac }\end{array}$ & $\begin{array}{l}1784 \\
1783 \\
1783 \\
1785 \\
1783 \\
1784 \\
1784\end{array}$ & $\begin{array}{l}\text { Erzieher im Waisenhaus der wal- } \\
\text { lonischen Kirche, Magdeburg } \\
\text { Kantor in Strasburg/Uckermark } \\
\text { Kantor in Frankfurt/Oder } \\
\text { Erzieher der Pensionäre } \\
\text { von Pfarrer Fort in Königsberg } \\
\text { Aufseher der Pensionäre } \\
\text { der École de Charité } \\
\text { Schreiblehrer an der École } \\
\text { de Charité } \\
\text { Erzieher im französischen } \\
\text { Waisenhaus Magdeburg }\end{array}$ \\
\hline 1783 & *Philippe Cordier & 1784 & Lehrer und Kantor in Schwedt \\
\hline 1784 & $\begin{array}{l}\text { *Jean Cazalét } \\
\text { Pierre Sarre }\end{array}$ & $\begin{array}{l}1784 \\
1784\end{array}$ & $\begin{array}{l}\text { Anstellung in Schlesien } \\
\text { Kantor und Lehrer } \\
\text { in Strasburg/Uckermark }\end{array}$ \\
\hline 1785 & $\begin{array}{l}\text { *Jacques Le Comte } \\
\text { *Jean Jacob Noé } \\
\text { *Pierre Francois } \\
\text { Sabouré }\end{array}$ & $\begin{array}{l}1789 \\
1786 \\
1789\end{array}$ & $\begin{array}{l}\text { Kirchenbediensteter in Berlin } \\
\text { Kantor und Lehrer in Wesel } \\
\text { Kirchenbediensteter in Berlin }\end{array}$ \\
\hline 1786 & *Pierre Mathieu & 1789 & Kirchenbediensteter in Berlin \\
\hline 1789 & Jean Jaques Valette & 1790 & Kantor in Klein-Ziethen \\
\hline 1794 & Samuel Boccard & 1794 & Kantor in Halle \\
\hline 1796 & $\begin{array}{l}\text { Guillaume Legrom } \\
\text { *Francois Provencal }\end{array}$ & $\begin{array}{l}1797 \\
1797\end{array}$ & $\begin{array}{l}\text { Kantor in Brandenburg } \\
\text { Studium am Französischen } \\
\text { Gymnasium in Berlin fortgesetzt }\end{array}$ \\
\hline 1798 & Samuel Chambeau & 1798 & Eintritt in das Predigerseminar \\
\hline 1799 & Henri Clément & 1801 & Kantor in Halberstadt \\
\hline 1800 & $\begin{array}{l}\text { Isaac Boccard } \\
\text { Henri Guyot } \\
\text { * Jean Lefevre }\end{array}$ & $\begin{array}{l}1804 \\
1804 \\
1804 \\
\end{array}$ & $\begin{array}{l}\text { Lehrer und Vertreter des Kantors } \\
\text { in Königsberg } \\
\text { Kantor in Neuhaldensleben } \\
\text { Kantor in Halberstadt }\end{array}$ \\
\hline 1805 & $\begin{array}{l}\text { Jean Abraham } \\
\text { Laurent }\end{array}$ & 1808 & Aufseher der École de Charité \\
\hline 1812 & $\begin{array}{l}\text { Jean Frederic } \\
\text { Lapierre } \\
\text { Guillaume Hurtienne }\end{array}$ & $\begin{array}{l}1817 \\
1815\end{array}$ & $\begin{array}{l}\text { Kantor in der Werderkirche } \\
\text { Anstellung beim Kaufmann } \\
\text { Arnous }\end{array}$ \\
\hline 1813 & $\begin{array}{l}\text { Jean Daniel Frederic } \\
\text { Blanbois }\end{array}$ & 1814 & Aufseher der École de Charité \\
\hline 1814 & Samuel Marot & 1815 & Aufseher der École de Charité \\
\hline
\end{tabular}

Trotz all dieser Bemühungen und vieler Erfolge konnten die hugenottischen Führungsgremien die politische Auflösung ihrer Kolonien und damit den Verlust ihrer Privilegien auf die Dauer nicht abwenden. Die Verwaltungsreformen des preußischen Staates von 1808/09 bestätigten die schlimmsten Befürchtungen der Hugenotten, selbst wenn sie sich einige Rechte zu sichern wußten - etwa 
weiterhin die Aufsicht über das französische Elementarschulwesen ausüben zu dürfen ${ }^{53}$. Zumindest aber war es gelungen, einem Teil des Nachwuchses seine Herkunft bewußt zu machen und in ihm den Wunsch zu wecken, diese Tradition fortzusetzen. In diesem Sinne soll abschließend ein Absolvent der Pépinière zu Wort kommen, Louis Albert Beauvais. Beauvais war 1818 in das Lehrerseminar aufgenommen worden und hatte dort eine vierjährige Ausbildung erhalten ${ }^{54}$. Anschließend hatte er eine Anstellung am hugenottischen Waisenhaus gefunden, wo man mit ihm so zufrieden war, daB er in der Festschrift anläßlich der Hundertjahrfeier der Anstalt (1825) wegen seiner Verdienste um die Erziehung der Zöglinge lobend erwähnt wurde ${ }^{55}$. Im Jahr 1828 wandte er sich schriftlich an die Direktoren des Hauses mit dem Vorschlag, eine Sonntagsschule für Lehrlinge einzurichten. Die Erziehungsanstalten der Hugenotten kümmerten sich nämlich über die Schulzeit hinaus um ihre Abgänger, indem sie ihnen Lehr- oder Dienstverhältnisse vermittelten. Besonders die Überwachung der Lehrlinge erwies sich aber als mühsam. Zum einen liefen die jungen Leute häufig aus der Lehre davon, zum anderen verloren sie bei deutschen Meistern ihre Französischkompetenz. Gerade dieser zweite Aspekt besorgte Beauvais, wie seine Argumentation für die Errichtung der Sonntagsschule zeigt:

Niemals [schreibt er einleitend] hat sich die Notwendigkeit, die französische Sprache wiederzubeleben, derart spüren lassen wie in unseren Tagen. Unsere Kirchen sind verlassen, kaum noch sieht man die Spuren der Brüderschaftsbande, die früher alle Kinder der französischen Refugierten wie eine einzige und gleiche Familie verbanden. Nicht mir kommt es zu, über die Mittel ins Detail zu gehen, mit denen man dem Geist der Nachfahren dieser Helden des Evangeliums [...] einen neuen Aufschwung geben und ein neues Leben einhauchen könnte. Heute möchte ich lhnen nur, meine Herren, für die zukünftigen Mitglieder unserer Kolonie, die in unseren frommen Stiftungen erzogen und mehr oder weniger im Französischen unterrichtet wurden, einen Weg weisen, wie die französische Sprache erhalten werden kann.

Nachdem er dieses Projekt vorgestellt hat, kommt Beauvais auf die Vorzüge der École de Dimanche zu sprechen, namentlich auf ihr Ziel, die "guten Sitten unter den Lehrlingen aufrechtzuerhalten« und diesen »die Geschichte ihrer noblen Herkunft« ins Herz zu pflanzen, die dann eine »feurige Liebe für ihre Religion« entflammen werde ${ }^{56}$.

${ }^{53}$ Dies freilich in Abhängigkeit von der Geistlichen und Schul-Deputation der Kurmärkischen Regierung. Vgl. die Kabinettsorder vom 03.02.1812, abgedruckt bei MURET, Brandenburg-Preußen (wie Anm. 33), S. 312; und AFrD (wie Anm. 16), Rep. 04-661, Bl. 4f. (27.02.1712).

${ }^{54}$ BFrD (wie Anm. 33), A-330 (Relation de l'École de Charité, 1818), S. 7: Eintritt; A-330 (Relation de l'École de Charité, 1822), S. 9: Austritt.

${ }^{55}$ Relation de la Maison d'Orphelins publiée à l'occasion de son jubilé centenaire celébré le 31. May 1825, Berlin 1825, S. 22.

${ }^{56}$ Das Originalzitat lautet im Zusammenhang: Jamais le besoin de faire revivre parmi nous la langue de nos pères ne s'est fait sentir davantage que de nos jours. Nos temples sont déserts et à peine voit on quelques traces de ce lien de fraternité qui jadis unissait tous les enfants 
Das überzeugte die Direktoren. Tatsächlich kam es 1829 zur Gründung der Ecole de Dimanche, ihre Leitung wurde Beauvais übertragen. Doch zeichnet sich an ihrem Geschick bereits der Untergang des französischen Bildungswesens in Berlin ab: Sie hatte nur ein Jahr Bestand ${ }^{57}$.

\section{Résumé français}

Selon le règlement des Églises réformées françaises, elles étaient obligées d'assurer l'enseignement de la jeunesse. Conformément à cette règle, les huguenots commencèrent directement après leur établissement dans le Brandebourg-Prusse à créer des centres d'éducation. Quelque temps après, toute communauté française possédait au moins une école élémentaire. A Berlin, où se trouvait la plus grande colonie sur le territoire des Hohenzollern, on créait par surcroît des établissements de formation approfondie - des centres d'enseignement supérieur, un lycée, un séminaire de prédicateurs. Le séminaire des professeurs huguenots, la Pépinière des Chantres et Mâtres d'École, fut fondé en 1779 par la Compagnie du Consistoire à Berlin. La cause de cette fondation était le manque croissant d'enseignants dont souffraient les écoles primaires françaises dans la capitale et à la campagne. La contrainte de fermer un nombre croissant d'écoles, qui résultait de cette situation, contrariait les efforts des comités directeurs huguenots de conserver l'indépendance de l'Église française et des colonies. Cette autonomie qui était basée sur des privilèges leur semblait dépendre surtout du maintien du français comme langue courante et culturelle. Comme un grand nombre de descendants des réfugiés entre-temps avait perdu la connaissance du français, la salle de classe française proposait un bon moyen de formation linguistique. En conséquence, dans le séminaire des professeurs, l'accent était mis sur l'enseignement de la religion et du français - pour le reste, il ne se distinguait guère des autres séminaires de professeurs contemporains qui étaient peu nombreux. Jusqu'en 1809, au moment où la Pépinière fut fermée pour la première fois, du moins temporairement, plusieurs douzaines d'étudiants avaient reçu en règle générale une formation de plusieurs années. Quelques-uns trouvaient par la suite un emploi dans une institution éducative française à Berlin ou dans le Brandebourg, parfois ils étaient même embauchés à Königsberg ou à Wesel. Certes, à long terme de tels succès ne pouvaient pas éviter la désintégration politique de ces colonies françaises, mais ils pouvaient retarder la disparition de leurs écoles longtemps après la réforme de l'État prussien qui eut lieu au début du $\mathrm{XIX}^{\mathrm{e}}$ siècle.

des refugiés français en une seule et même famille. Il ne m'appartient pas d'entrer dans de grands détails sur les moyens qu'il faudrait mettre en usage pour donner un nouvel élan et prêter une nouvelle vie à l'esprit des descendants de ces héros de l'Evangile [...], je ne veux aujourd'hui, Messieurs, Vous entretenir que d'un moyen de conserver la langue française, applicable à ceux des membres futurs de notre colonie, qui, élevés dans nos fondations pieuses, instruits plus ou moins dans le français, sont placés ensuite, la plupart chez des maîtres allemands, oublient, pendant les quatre ou cinq années que dure leur apprentissage, tout ce qui [sic] peuvent avoir appris de leur langue. [...] Outre la conservation de la langue française il y auroit encore un autre avantage [...]. Le tuteur chargé de cette école pourra [pourvoir] en même temps au maintien des bons mours des apprentis [...]. Ils trouveraient en lui un conseiller, un ami, un père et ainsi ils s'empresseront à profiter des leçons qu'on leur donnera. L'histoire de leur noble origine, et des sacrifices multiples dont ils ont été les objets ferait revivre dans leurs jeunes cours l'esprit de leurs pères et leur inspirerait à tous un amour ardent pour leur religion, AFrD (wie Anm. 16), Rep. 04-903, Bl. $90 \mathrm{f}$.

${ }^{57}$ Zur École de Dimanche vgl. MURET, Brandenburg-Preußen (wie Anm. 33), S. 163f. 